\title{
Interplay between depressive-like behavior and the immune system in an animal model of prenatal dexamethasone administration
}

\section{Susana Roque, Tiago Gil Oliveira, Claudia Nobrega, Palmira Barreira-Silva, Cláudio Nunes-Alves, Nuno Sousa, Joana Almeida Palha and Margarida Correia-Neves*}

Life and Health Sciences Research Institute (ICVS), School of Health Sciences, University of Minho, Braga, Portugal

\section{Edited by:}

Carmen Sandi, École Polytechnique

Fédérale de Lausanne, Switzerland

Reviewed by:

Francesca Cirulli, Istituto Superiore di Sanità, Italy

Muriel Darnaudery, University of Lille 1, France

\section{*Correspondence:}

Margarida Correia-Neves, Life and Health Sciences Research Institute, School of Health Sciences, University of Minho, Campus de Gualtar,

4710-057 Braga, Portugal.

e-mail:mcorreianeves@ecsaude.

uminho.pt
Glucocorticoids, namely dexamethasone, are prescribed during late gestation in pregnancies at risk of originating premature newborns, to promote fetal lung maturation. However, adverse early life events have been reported to induce long-lasting changes in the immune and central nervous systems. The accumulating evidence on bidirectional interactions between both systems in psychiatric disorders like depression, prompted us to further investigate the long-term impact of prenatal dexamethasone administration in depressive-like behavior, the immune system and in the ability to mount an immune response to acute infection. The adult male offspring of pregnant dams treated with dexamethasone present depressive-like behavior concomitant with a decrease in $\mathrm{CD}^{+} \mathrm{T}$ lymphocytes and an increase in $\mathrm{B}$ and $\mathrm{CD} 4^{+}$regulatory $\mathrm{T}$ cells. This is accompanied by lower levels of serum interleukin-6 (IL-6) and IL-10. Despite of these differences, when spleen cells are stimulated, in vitro, with lipopolysaccharide, those from adult rats prenatally treated with dexamethasone display a stronger pro-inflammatory cytokine response. However, this immune system profile does not hamper the ability of rats prenatally treated with dexamethasone to respond to acute infection by Listeria monocytogenes. Of notice, L. monocytogenes infection triggers depressive-like behavior in control animals but does not worsen that already present in dexamethasone-treated animals. In summary, prenatal administration of dexamethasone has long-lasting effects on the immune system and on behavior, which are not further aggravated by acute infection with L. monocytogenes.

Keywords: prenatal, dexamethasone, immunity, behavior, depression, Listeria monocytogenes

\section{INTRODUCTION}

Observations from the last decades have revealed an interplay between the immune and the central nervous systems in mood disorders like depression (Miller, 2010). On one hand, depressed patients frequently show alterations in their immune system and, on the other, inflammatory disorders or immunotherapies involving pro-inflammatory cytokines (mainly IL-2, TNF, and IFN- $\alpha$ ), have been associated with depressive symptoms (Miller et al., 2009).

In parallel, in recent years, a considerable amount of evidence has showed that early life events can trigger programming effects in both the nervous and the immune systems. One of the best well-known programmers is the glucocorticoid dexamethasone, commonly administered to pregnant women at risk of premature delivery, and to premature neonates, in order to promote lung maturation (Trautman et al., 1995; Hirvikoski et al., 2007; Karemaker et al., 2008; Hofmeyr, 2009; Mesquita et al., 2009). In fact, prematurely born children neonatally treated with dexamethasone have, at school age, significantly lower plasma cortisol levels during and after exposure to a psychosocial stress test when compared to prematurely born non-treated children (Karemaker et al., 2008). This observation suggests that the activity of the hypothalamicpituitary-adrenal (HPA) axis is influenced by early treatment with dexamethasone. Of notice, this alteration is accompanied by an imbalanced cytokine profile. Specifically, production of IFN- $\gamma$ upon stimulation of T cells with anti-CD2/CD28 monoclonal antibodies is significantly higher in children neonatally treated with dexamethasone (Karemaker et al., 2008).

Several preclinical studies have also assessed the impact of prenatal glucocorticoid exposure. On the central nervous system side, it has been associated with disruption of the HPA axis and with alterations on cognitive and motor development (Trautman et al., 1995; Welberg and Seckl, 2001; Coe and Lubach, 2005; Hirvikoski et al., 2007; Mesquita et al., 2009). Furthermore it predisposes adult animals to anxiety and to depressive-like behavior after chronic mild stress in adulthood (Oliveira et al., 2006). However, the window of prenatal dexamethasone treatment seems to be crucial for the induction of long-lasting effects on the behavior in adulthood. In fact, dexamethasone treatment of rats, during the 3 weeks of gestation, does not induce alterations in the forced swim test (FST), while the same protocol administered only in the last week of gestation reduces the immobility time in the FST (Welberg and Seckl, 2001). Of interest, prenatal dexamethasone treatment leads to reduced volumes and cell number in the nucleus accumbens, which is a brain region strongly correlated with a reduced rate of cell proliferation (Leao et al., 2007) and is suggested to predispose to addictive behavior (Rodrigues et al., 2010). On the immune system side, early 
life treatment with dexamethasone in animal models consistently suggests a long-term impact on the immune function, namely in the structure of the thymus (Dietert et al., 2003), T cell proliferation (Coe and Lubach, 2000), cytokine production (Bakker et al., 2000; Dietert et al., 2003), and in the predisposition to autoimmune disorders (Bakker et al., 2000), even though the results are not always concordant. Curiously, although several studies demonstrate that prenatal administration of dexamethasone impacts on the immune and central nervous systems, the specific effects are quite discordant between studies, probably due to differences in the administration period (during pregnancy or soon after birth) or dosage, and also in the age in which the animals are analyzed.

Given the World Health Organization's advice that corticosteroid therapy should be incorporated into comprehensive maternal health-care services, and included in official guidelines for maternity care (Hofmeyr, 2009), it is crucial to understand its potential long-term impact in the immune and central nervous systems and on their interplay. A better perspective of the full spectrum of effects triggered by glucocorticoid therapy during pregnancy requires a combined multimodal approach, in the same animal model, that assesses the impact in the immune system and in behavioral alterations, which is the focus of the present study.

\section{MATERIALS AND METHODS}

\section{ANIMALS AND DEXAMETHASONE ADMINISTRATION}

Female Wistar rats with 14 days of pregnancy were purchased from Charles River Laboratories (Barcelona, Spain) and individually housed. Subcutaneous injections of dexamethasone (1 mg/ $\mathrm{kg}$ ) or saline were administered on pregnancy days 18 and 19 (Oliveira et al., 2006, 2010; Leao et al., 2007). For each experiment 12 pregnant dams were used ( 6 for dexamethasone treatment and 6 controls with saline). The male progeny was separated on postnatal day 21 and housed according to the prenatal treatment (Dexa or Sal) in groups of two to three animals per cage from the same litter. Each group (Sal or Dexa) was constituted with animals from distinct litters (no more than three animals from each litter per experimental group). All animals were housed in an animal facility at $22^{\circ} \mathrm{C}$, relative humidity of $55 \%$, in a $12 \mathrm{~h}$ light $/ 12 \mathrm{~h}$ dark cycle, with food and sterile tap water available ad libitum. The experimental procedures were performed at 3 months of age. The biometric parameter assessed were body spleen, thymus, and adrenal glands weights (at sacrifice). Blood from sacrificed rats was allowed to clot and the serum, collected after centrifugation, was frozen at $-80^{\circ} \mathrm{C}$ until use. All animal experiments were performed in accordance with National and European Commission guidelines for the care and handling of laboratory animals and were approved by the National Veterinary Directorate and by the local Animal Ethical Committee.

\section{FLOW CYTOMETRY}

To evaluate the proportion of the distinct populations of the immune system, flow cytometry was performed using single-cell suspensions from spleen and thymus. Splenic erythrocytes were depleted by incubation with a hemolytic solution $\left(155 \mathrm{mM} \mathrm{NH}_{4} \mathrm{Cl}\right.$, $10 \mathrm{mM} \mathrm{KHCO}_{3}, \mathrm{pH} \mathrm{7.2)}$ for $5 \mathrm{~min}$ at room temperature. For cell surface staining, $5 \times 10^{5}$ cells were used (in the case of Foxp 3 staining, $10^{6}$ cells) from each individual rat and incubated with specific antibodies (Table 1) for $20 \mathrm{~min}$ on ice. Cell surface markers were analyzed using specific antibodies for CD11 b/c (OX-42), CD45RA (OX-33; Caltag, CA, USA), CD161 (10/78), CD25 (OX-39), CD4 (W3/25), CD8 (G28; BioLegend, San Diego, CA, USA), granulocytes (RP-1; BD Pharmingen, San Diego, CA, USA), Foxp3 (FJK-16a), CD3 (G4.18; eBioscience, San Diego, CA, USA). All cells were fixed with $2 \%$ formaldehyde after staining. A total of 50,000 events were acquired on a FACSCalibur flow cytometer (Becton Dickinson, NJ, USA) using the Cell Quest software (Becton Dickinson, NJ, USA); analysis of the cell populations was performed using the FlowJo software (Tree Star, Ashland, OR, USA).

\section{MULTIPLEX CYTOKINE ANALYSIS}

The concentration of a panel of cytokines (IL- $1 \beta$, IL-6, IFN- $\gamma$, IL-4, IL-10) was measured both in serum and in spleen cells supernatants stimulated in vitro with lipopolysaccharide (LPS, a component of the cell wall of Gram-negative bacteria) or with no stimulus, using a rat Bio-Plex cytokine assay (Bio-Rad, Hercules, CA, USA). The rat Bio-Plex cytokine assay was performed according to the manufacturer instructions. The sensitivity ranges were of $11.80 \mathrm{pg} / \mathrm{mL}$ for IL-1 $\beta, 0.11 \mathrm{pg} / \mathrm{mL}$ for IL-4, $0.93 \mathrm{pg} / \mathrm{mL}$ for IL-6, $3.44 \mathrm{pg} / \mathrm{mL}$ for IL-10, and $0.30 \mathrm{pg} / \mathrm{mL}$ for IFN- $\gamma$.

\section{IN VITRO STIMULATION OF SPLEEN CELLS}

Spleen cells (prepared as described previously), resuspended in DMEM (supplemented with 10\% heat inactivated FCS, $10 \mathrm{mM}$ HEPES buffer, $1 \mathrm{mM}$ sodium pyruvate, $2 \mathrm{mM}$ L-glutamine, $50 \mu \mathrm{g} /$ $\mathrm{mL}$ streptomycin, and $50 \mathrm{U} / \mathrm{mL}$ penicillin, all from Invitrogen, CA, USA), were distributed into 96 -well plates $\left(5 \times 10^{5}\right.$ cells/well $)$, in triplicate wells, and incubated with or without $5 \mu \mathrm{g} / \mathrm{ml} \mathrm{LPS} \mathrm{(Escherichia}$ coli, serotype O26:B6; Sigma, St Louis, USA). Supernatants were collected after $48 \mathrm{~h}$ incubation at $37^{\circ} \mathrm{C}, 5 \% \mathrm{CO}_{2}$ atmosphere, and frozen at $-80^{\circ} \mathrm{C}$ until use.

\section{RAT INFECTION AND OUANTIFICATION OF ORGAN BACTERIAL LOADS}

At 3 months of age rats were intravenously infected through a lateral tail vein with $10^{6}$ colony-forming units (CFU) of Listeria monocytogenes EGDe (provided by Dr. S. Sousa and Dr. D. Cabanes, Instituto de Biologia Molecular e Celular, Porto, Portugal). We performed preliminary experiments and confirmed that the wellestablished protocol of L. monocytogenes infection (Goettsch et al.,

Table 1 | Antibodies used for identification of different spleen cells.

\begin{tabular}{ll}
\hline Immune cells & Phenotype \\
\hline $\mathrm{CD} 4^{+}$T cells & $\mathrm{CD} 3^{+} \mathrm{CD} 4^{+}$(gated lymphocytes in FSC, SSC) \\
$\mathrm{CD} 8^{+}$T cells & $\mathrm{CD} 3^{+} \mathrm{CD} 8^{+}$(gated lymphocytes in FSC, SSC) \\
$\mathrm{CD} 4^{+}$regulatory T cells & $\mathrm{CD} 3^{+} \mathrm{CD} 4^{+} \mathrm{CD} 25^{+}$Foxp3 $^{+}$(gated lymphocytes \\
& in FSC, SSC) \\
NKT cells & $\mathrm{CD} 3^{+} \mathrm{CD} 161^{+}$ \\
B cells & $\mathrm{CD} 45 \mathrm{Ra}^{+}$(gated lymphocytes in FSC, SSC) \\
Macrophages & $\mathrm{CD} 11 \mathrm{bc}{ }^{+}$(gated in all cells except granulocytes \\
& in FSC, SSC) \\
Neutrophils & $\mathrm{CD} 11 \mathrm{bc} \mathrm{C}^{+}$granulocytes (gated granulocytes in $^{+}$ \\
NK cells & $\mathrm{FSC}, \mathrm{SSC})$
\end{tabular}


1996; Cabanes et al., 2008) is replicated in rats. At days 2, 4, and 8 post infection rats were sacrificed and spleen and liver were aseptically collected for bacterial load evaluation. Briefly, organs were homogenized, serially diluted in ice-cold water and plated onto Brain Heart Infusion medium (BHI; Laboratorios Conda, Spain). The plates were incubated for 1 day at $37^{\circ} \mathrm{C}$ and the number of CFU counted.

\section{FORCED SWIMMING TEST}

Learned helplessness was evaluated in the FST. This protocol was performed in non-infected animals and 4 days after infection with L. monocytogenes, as previously described (Goncalves et al., 2008). Briefly, a pre-test session was performed by placing the rats for $5 \mathrm{~min}$ in cylinders filled with water $\left(24 \pm 1^{\circ} \mathrm{C}\right)$, depth enough so they could not support themselves by placing the paws or tail on the base of the cylinders. After $24 \mathrm{~h}$ animals were tested during $5 \mathrm{~min}$. Only the second session was recorded and later scored by two independent researchers who were blind to the experimental conditions. Immobility time and latency to immobility were computed and used as a measure of depressive-like behavior.

\section{SUCROSE PREFERENCE TEST}

Anhedonic behavior was assessed by the sucrose preference test (SPT) as described previously (Bessa et al., 2009). Briefly, all animals were food and water deprived for $24 \mathrm{~h}$ and exposed afterward to two bottles either with $1 \%$ of sucrose solution or with sterile tap water for a period of $1 \mathrm{~h}$. To avoid possible effects of side preference in drinking behavior, the position of the bottles was switched in each trial and the test was performed three times in a period of 2 weeks. Sucrose preference was calculated as the percentage of consumed sucrose solution of the total of liquid drunk.

\section{STATISTICAL ANALYSIS}

All data are represented as mean + SEM. Data were verified for Gaussian distribution. Student's $t$ test was used to calculate differences between the Sal and Dexa groups. For the analysis of cytokine production upon LPS stimulation, statistical analysis was performed with the non-parametric Mann-Whitney $U$ test. The impact of L. monocytogenes on cell types, cytokines levels and CFU after 2, 4, and 8 days of infection were analyzed with two-way ANOVA. Significance is referred as $*$ for $P<0.05$.

\section{RESULTS}

PRENATAL DEXAMETHASONE TREATMENT INDUCES DEPRESSIVE-LIKE BEHAVIOR AND ALTERATIONS IN THE ADULT IMMUNE SYSTEM

Prenatal dexamethasone treatment did not impact on litter size nor on the sex of the progeny since pregnant rats injected with saline (Sal) or dexamethasone (Dexa) had an average of 10 rats per litter with a 50\% average of male and female. The progeny of dexamethasone-treated dams presented, as expected, reduced body weight at birth, which remained significantly reduced at 60 days of age (Oliveira et al., 2006), but no longer at 3 months when body weight was identical in both treatment groups (Figure 1).

To investigate the impact of prenatal administration of dexamethasone on behavior and on the immune system we first analyzed the depressive-like behavior of rats in the FST and SPT, at 3 months of age. When compared to prenatally vehicle-injected rats, animals prenatally treated with dexamethasone showed an increased immobility time $(t=2.4, P=0.02)$ as well as a trend for decreased latency to immobility time in the FST (Figure 2A). Accordingly, a reduced hedonic behavior was also observed in the Dexa animals since they presented a decrease in the sucrose preference (Figure $2 \mathbf{B}$ ) when compared with Sal rats $(t=2.9, P=0.0065)$.

As our protocol of dexamethasone prenatal administration causes a disruption on the HPA axis (Oliveira et al., 2006), we determined biometric parameters known to be influenced by altered corticosteroid levels. Even though changes in body, spleen, and adrenal glands weight were not observed, we found a decreased thymus weight $(t=2.4, P=0.02)$ in Dexa rats (Figure 1). The thymus, in particular the $\mathrm{CD} 4^{+} \mathrm{CD} 8^{+}$thymocyte population, is extremely sensitive to corticosteroids (Ashwell et al., 2000). Therefore, we evaluated the impact of prenatal dexamethasone administration on the four main thymic cell populations: double negative $\left(\mathrm{CD} 4^{-} \mathrm{CD} 8^{-}\right)$, double positive $\left(\mathrm{CD} 4^{+} \mathrm{CD} 8^{+}\right), \mathrm{CD} 4$ single positive $\left(\mathrm{CD}^{+} \mathrm{CD}^{+} \mathrm{CD}^{-}\right)$, and $\mathrm{CD} 8$ single positive $\left(\mathrm{CD} 3^{+} \mathrm{CD} 4^{-} \mathrm{CD} 8^{+}\right)$. Interestingly, despite the decrease in thymus weight, no alterations were observed in the proportions of the four main thymic cell populations (Figure 3A).
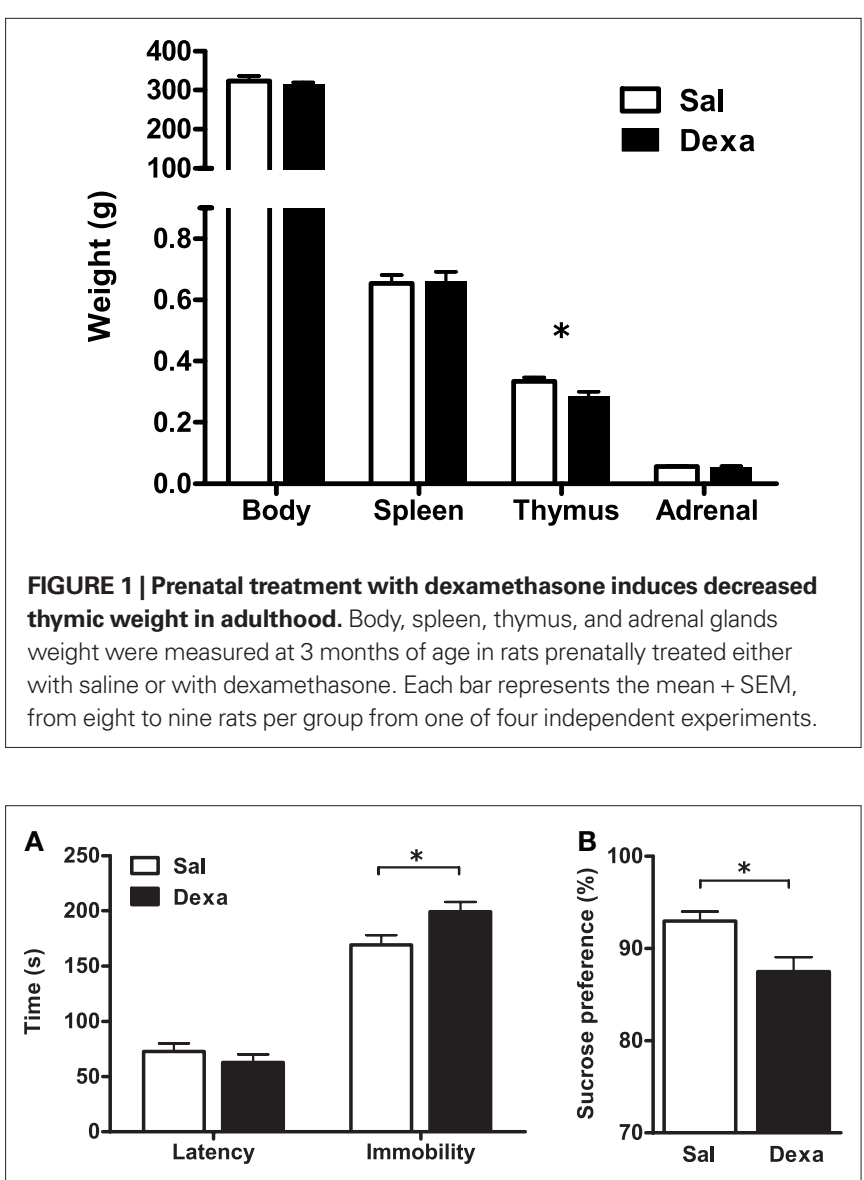

FIGURE 2 | Prenatal administration of dexamethasone induces depressive-like behavior. (A) Latency to first immobility and duration of immobility in the forced swim test and (B) sucrose preference were assessed in Sal and Dexa rats. Each bar represents the mean + SEM from 16 to 17 rats per group. Data from SPT are representative from three independent trials. 

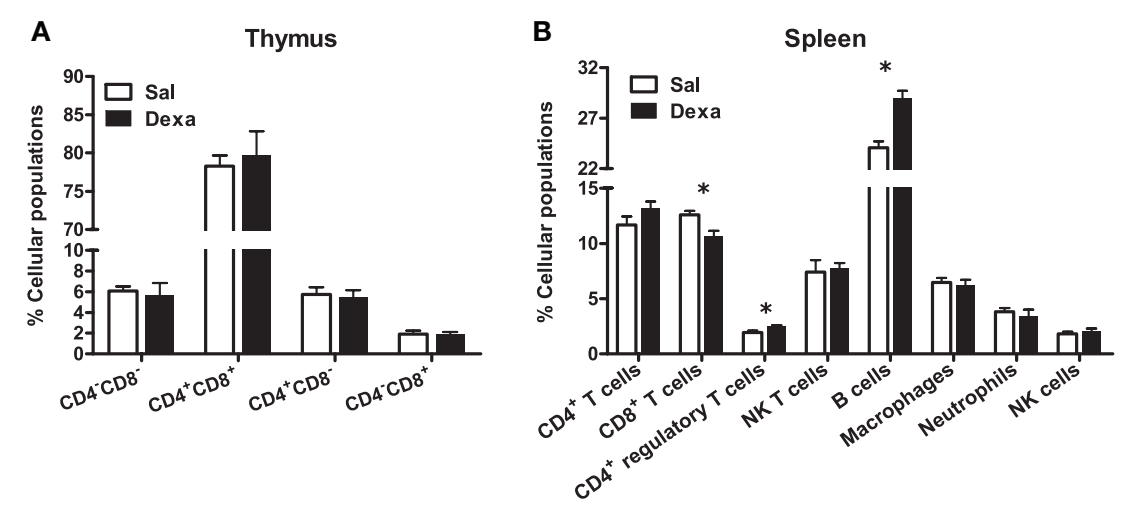

FIGURE 3 | Prenatal dexamethasone treatment induces, in the adult progeny, alterations in splenic $\mathrm{CD8}^{+}$and $\mathrm{CD}^{+}$regulatory $\mathrm{T}$ and on $\mathrm{B}$ cells but not in the proportion of the four main thymic cell populations. (A) Thymocytes from Sal and Dexa groups were stained with specific antibodies and analyzed by flow cytometry. Data are represented as the mean + SEM, from eight rats per group from one of four independent experiments. (B) Splenocytes from Sal and Dexa groups were stained with specific antibodies and analyzed by flow cytometry. Each bar represents the mean + SEM from 9 to 12 animals per group from one of three independent experiments.
To further explore the immune system, we subsequently studied the immune cell populations present in the spleen of Dexa and Sal rats. Prenatal treatment with dexamethasone induced, in adulthood, a decrease in $\mathrm{CD}^{+} \mathrm{T}$ cells $(t=2.9, P=0.01)$ and an increase in both $\mathrm{B}(t=4.7, P=0.0004)$ and $\mathrm{CD} 4^{+}$regulatory T cells $(t=3$, $P=0.006$ ) in the spleen (Figure 3B). No differences were observed in the percentage of total $\mathrm{CD}^{+}$and NK T cells, macrophages, neutrophils or in NK cells of Dexa rats when compared to Sal animals (Figure 3B). Interestingly, we searched for correlations between the behavior in the FST and SPT and the percentage of immune cells in the spleen and only a negative correlation between latency time of the FST and the percentage of $\mathrm{CD}^{+} \mathrm{T}$ cells $(r=-0.8089$, $P=0.0003$ ) was observed. Moreover an almost significant negative correlation between the percentage of B cells and latency time in FST $(r=0.4893, P=0.0542)$ was also present.

Finally, we measured the levels of cytokines, which are key players in the communication between the immune and the central nervous systems known to be involved in the pathology of mood disorders (Miller et al., 2009). In basal conditions, Dexa rats presented an overall reduction in the serum levels of the cytokines analyzed (IL-1 $\beta$, IL-6, IFN- $\gamma$, IL-4, IL-10) when compared with Sal (Figure 4); however, only the reductions in IL-6 $(t=2.7, P=0.01)$ and IL-10 $(t=2.3, P=0.03$; a pro- and an anti-inflammatory cytokine, respectively) reached statistical significance (Figure 4).

\section{DEXA RATS SHOW AN INCREASED PRO-INFLAMMATORY PROFILE AFTER IN VITRO LPS STIMULATION BUT NO DIFFERENCES IN DEPRESSIVE-LIKE BEHAVIOR OR IN THE IMMUNE RESPONSE TO LISTERIA MONOCYTOGENES INFECTION}

Since we found that prenatal dexamethasone treatment induces, in adulthood, alterations in spleen immune cell populations and in the serum cytokine profile, we next asked how the immune system react to external stimuli and how would this impact on depressive-like behavior. For that, since Dexa rats present an increase in the percentage of B cells and LPS is a B cell mitogen (Sultzer and Goodman, 1976), we first stimulated splenocytes with LPS and analyzed the production of a panel of cytokines. Upon in vitro stimulation with LPS, spleen cells from Dexa rats displayed increased production of
IL-6 when compared with cells from the Sal group $(U=51, P=0.002$; Figure 5), thus showing an exacerbated pro-inflammatory response to this inflammatory stimulus. No differences were observed in the production of IL-1 $\beta$, IFN- $\gamma$, IL-4, or IL-10 (Figure 5).

In addition, we studied the impact of an in vivo acute infection in the immune response and behavior. We chose to test the immune response to the acute L. monocytogenes intracellular infection, as this response is mainly mediated by $\mathrm{CD}^{+} \mathrm{T}$ cells (Pamer, 2004), which we found to be decreased in Dexa rats. Interestingly, at 2, 4, and 8 days post infection no differences were observed in body, spleen, thymus, or adrenal glands weight (data not shown). Moreover, the alterations in non-infected Dexa rats spleen cell populations (Figure 3B) disappeared upon infection with L. monocytogenes (Figure 6). In fact, during the course of infection (2, 4 , and 8 days), no major differences were observed in total $\mathrm{CD}^{+}, \mathrm{CD}^{+}, \mathrm{CD} 4^{+}$regulatory and NK T cells, B cells, macrophages, neutrophils or NK cells between Sal and Dexa rats (Figure 6). However, two-way ANOVA showed a significant effect of time in the percentage of $\mathrm{CD}^{+}\left(F_{2,36}=35.8\right.$, $P<0.0001), \mathrm{CD}^{+}$regulatory $\left(F_{2,36}=11, P=0.0002\right), \mathrm{NK}\left(F_{2,37}=74.7\right.$, $P<0.0001)$ T cells, macrophages $\left(F_{2,37}=7.6, P=0.002\right)$, neutrophils $\left(F_{2,36}=13.9, P<0.0001\right)$, and NK cells $\left(F_{2,36}=36.1, P<0.0001\right)$. Time of infection also impacted on the levels of IL-6 $\left(F_{3,38}=3.06\right.$, $P=0.0398)$, IL-10 $\left(F_{3,38}=12.38, P<0.0001\right)$.

No differences between Sal and Dexa groups were found in the serum levels of IL- $1 \beta$, IL- 6 , IFN- $\gamma$, IL-4, and IL- 10 at any time point of infection (2, 4, and 8 days; Figure 7). In accordance, the evaluation of the bacterial load revealed identical susceptibility to acute infection with L. monocytogenes (Figure 8). Altogether these results showed that Dexa rats can mount an appropriate immune response to this particular infection.

In parallel, we assessed depressive-like behavior at 4 days upon infection. In the FST, as previously observed (Figure 2), noninfected Dexa rats presented a decreased latency time $(t=2.9$, $P=0.01)$ and increased immobility time $(t=2.3, P=0.03)$ when compared to Sal (Figure 9). Curiously, 4 days after infection these differences disappeared (Figure 9), as infection triggered, in Sal rats, an increase in immobility time almost statistical significant $(t=2.1$, $P=0.06)$ when compared to Sal non-infected rats (Figure 9). 

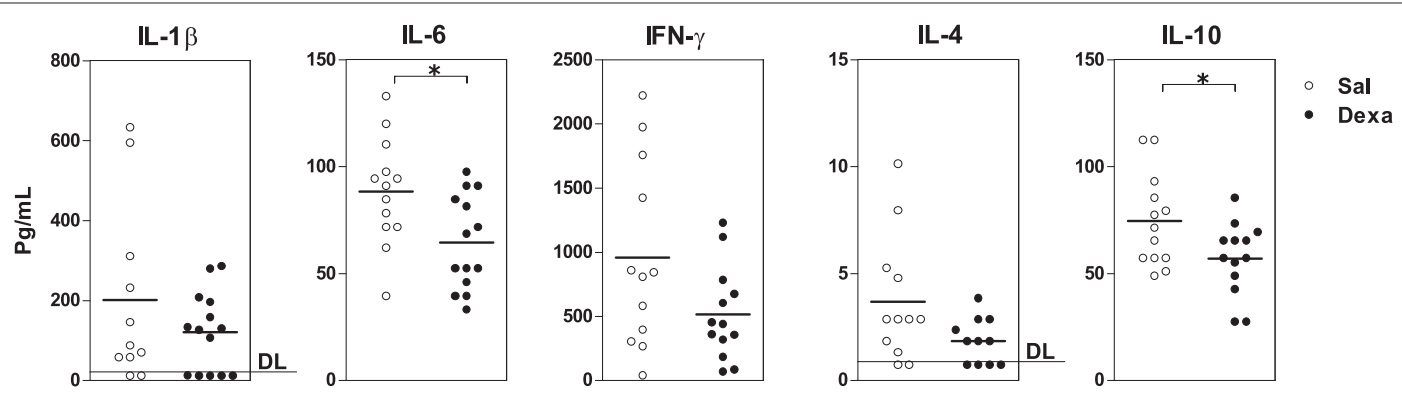

FIGURE 4 | Prenatal treatment with dexamethasone induces a decrease in adult serum IL-6 and IL-10 levels. Serum IL-1 $\beta$, IL-6, IFN- $\gamma$, IL-4, and IL-10 concentrations were determined using a multiplex cytokine assay. Each point represents one rat and the line corresponds to the mean of $12-13$ rats per group from two independent experiments. DL, detection limit.
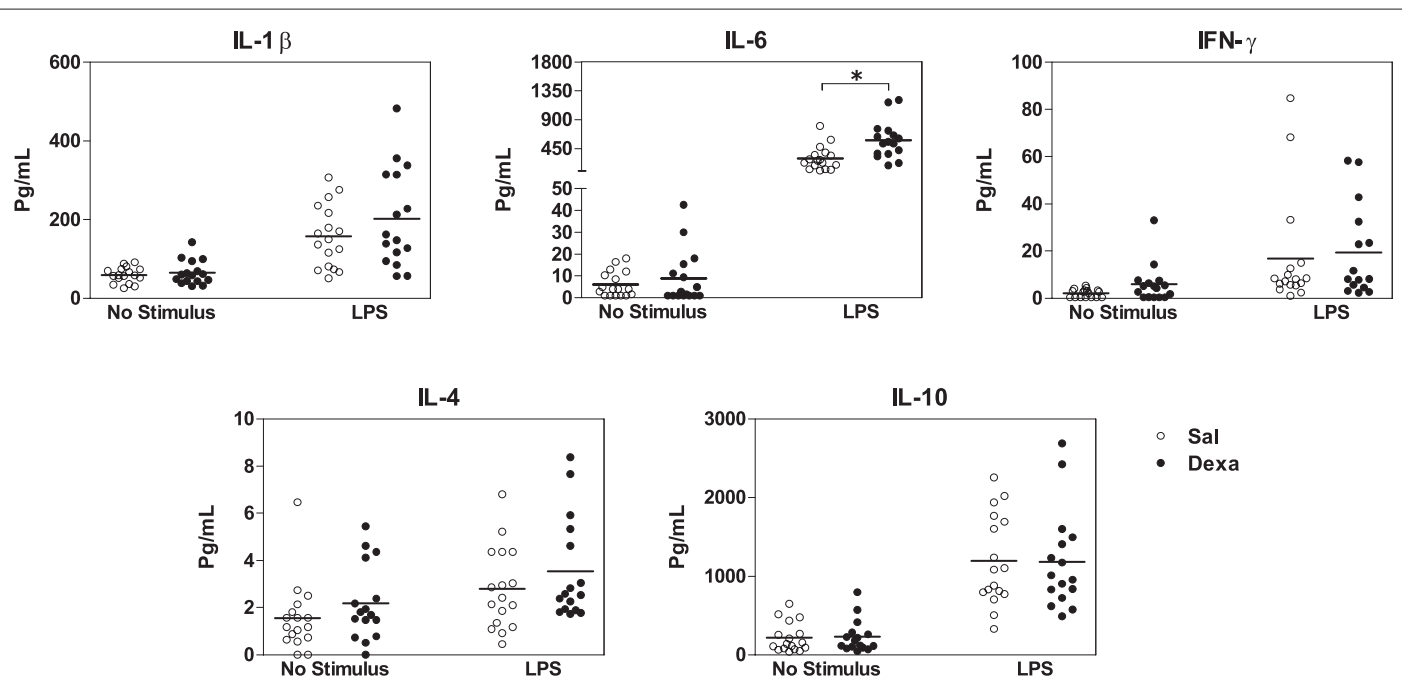

FIGURE 5 | Splenocytes from Dexa rats produce increased levels of IL-6 after LPS stimulation. Spleen cells from Sal and Dexa rats were stimulated with LPS $(5 \mu \mathrm{g} / \mathrm{mL})$ during $48 \mathrm{~h}$. Cytokine concentrations were determined by a multiplex cytokine assay in culture supernatants. Each point represents one rat and the line corresponds to the mean of 12-13 rats per group from two independent experiments.

\section{DISCUSSION}

The present study combined a behavioral and an immunological assessment of rats prenatally exposed to dexamethasone. The data collected show that these animals, when adults, display a depressivelike phenotype that is associated with decreases in IL-6 and IL-10 serum levels and with alterations in spleen immune cell populations (decrease in the percentage of $\mathrm{CD} 8^{+} \mathrm{T}$ cells and increase in regulatory $\mathrm{CD} 4^{+} \mathrm{T}$ and $\mathrm{B}$ cells). Moreover, spleen cells of Dexa rats display, after stimulation with LPS, an increased pro-inflammatory profile of cytokine production. However, rats treated with dexamethasone during the prenatal period do not show alterations in the susceptibility to acute infection with L. monocytogenes. Interestingly, this infection does not aggravate the depressive-like phenotype already present in non-infected Dexa animals.

The rat model used in this study has previously been shown to have disruption on the HPA axis (Oliveira et al., 2006). Thus, it is not surprising that prenatal dexamethasone treatment was able to induce depressive-like behavior (decrease latency time and increase immobility time in the FST and decreased sucrose preference) in 3 months old rats. Accordingly, increased immobility in the FST was also observed by others, after dexamethasone prenatal treatment (Hauser et al., 2009). The fact that Oliveira et al. (2006) did not observe altered behavior in the FST is likely related with the age of the animals, studied at 8 months; as has also been shown in the case of anxious-like behavior (present at 2 months and no longer at 8 months of age).

In the present study we extend the link of depressive-like phenotype observed in this animal model (increased immobility in the FST and decreased sucrose preference) to alterations in the immune system that may be linked to HPA dysregulation. In fact, HPA axis alterations are described to impact in the immune system homeostasis. As an illustration, the high sensitivity of the thymus to corticosteroids, given the expression of glucocorticoid receptors in thymic cells throughout life, is well-established (Ashwell et al., 2000). We now confirmed a sustained decrease in the thymus weight of adult rats treated prenatally with dexamethasone, even though no alterations were observed in the basal levels of corticosterone (data not shown). Others have shown that dexamethasone administration during the two last thirds of gestation leads to a marked alteration in the thymus structure in juvenile (5 weeks of age) and in adults rats 

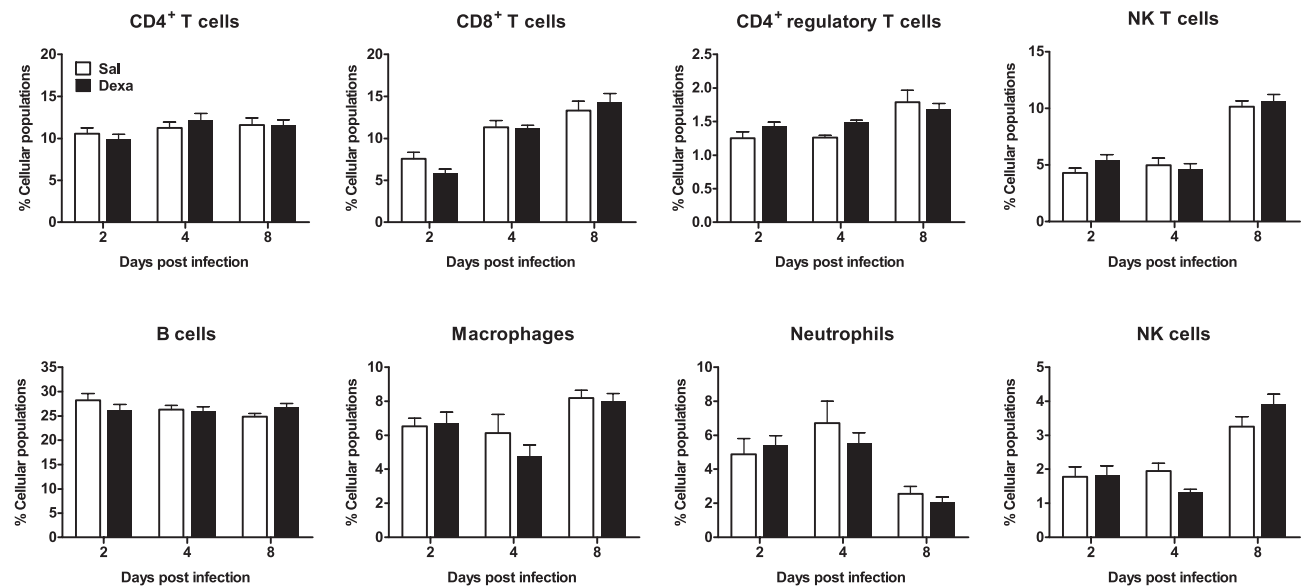

FIGURE 6 | Infection with L. monocytogenes results in a similar percentage of spleen cell populations in Sal and Dexa rats. Two, four, and eight days after L. monocytogenes infection, spleen cells from Sal and Dexa groups were stained with specific antibodies and analyzed by flow cytometry. Each bar represents the mean + SEM from six to eight animals per group.
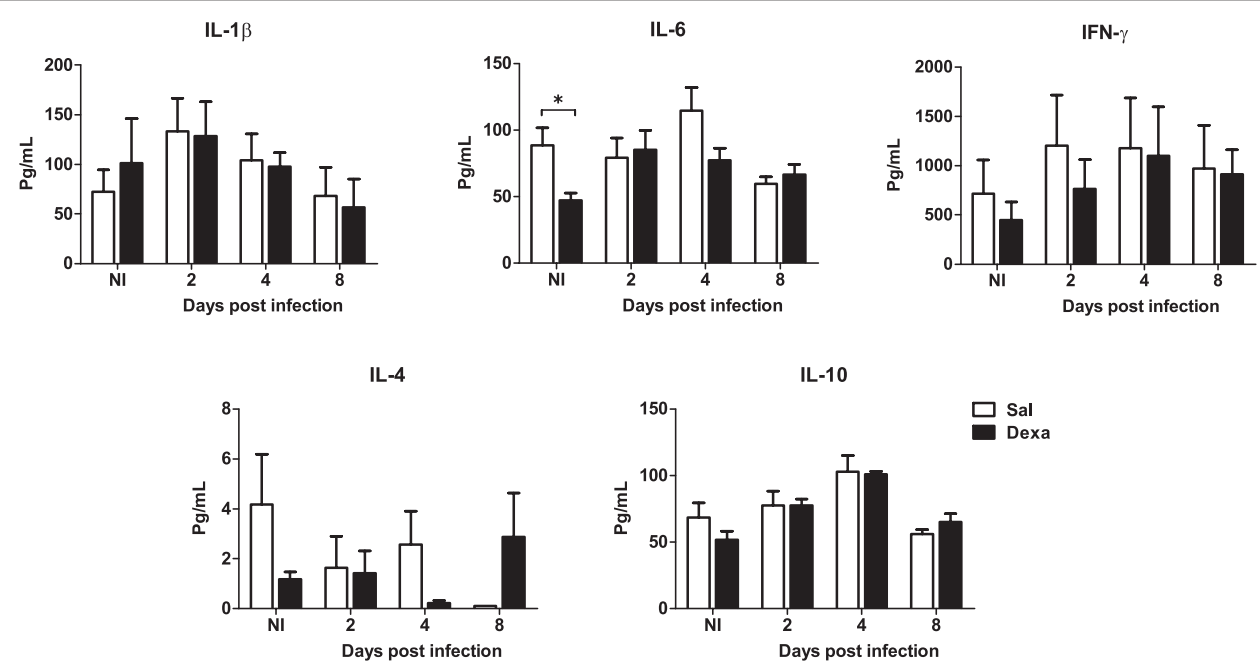

FIGURE 7 |Adult rats prenatally treated with Dexa or with Sal present a similar serum cytokine profile after $L$. monocytogenes. IL-1 $\beta$, IL-6, IFN- $\gamma$, IL-4, and IL-10 production upon infection was determined measuring their serum concentration with a multiplex cytokine assay. Each point represents one rat and the line corresponds to the mean of six to eight rats per group.

(13 weeks of age; Dietert et al., 2003). Interestingly, in adulthood, we found that the proportion of all major thymic cell populations was unaffected, which fits previous observation in a similar model (prenatal treatment of dexamethasone $400 \mu \mathrm{g}$, intraperitoneally, on days 17 and 19 of gestation; Bakker et al., 1995). However, it should be noticed that this adulthood stability was preceded by significant alterations in the proportion of thymocyte populations (Bakker et al., 1995). More importantly, there is evidence that corticosteroids are involved in thymocyte selection, as neonatal treatment with dexamethasone was reported to induce alteration in the T cell receptor $\mathrm{V} \beta$ repertoire (Bakker et al., 2001); this may cause longlasting alterations in the $\mathrm{T}$ cell repertoire and in the susceptibility to $\mathrm{T}$ cell related-disorders.
In line with the above mentioned hypothesis, we show herein, for the first time, alterations in the homeostasis of the spleen cells in adult animals prenatally treated with glucocorticoids. In fact, we found a decrease in the percentage of $\mathrm{CD}^{+} \mathrm{T}$ cells in the spleen of Dexa animals; yet, we cannot at this point ascribe these changes to thymic $\mathrm{T}$ cell differentiation, namely because there were no changes in the proportion of major thymic cell populations. Additionally, prenatal dexamethasone treatment also increased the percentage of $\mathrm{CD} 4^{+}$regulatory T cells $\left(\mathrm{CD} 4^{+} \mathrm{CD} 25^{+}\right.$ Foxp $3^{+}$). Interestingly, previous studies have shown that in vitro incubation of human $\mathrm{CD}^{+} \mathrm{T}$ cells with dexamethasone leads to upregulation of the mRNA levels of Foxp3 (Karagiannidis et al., 2004). This suggests an interaction between corticosteroids and 

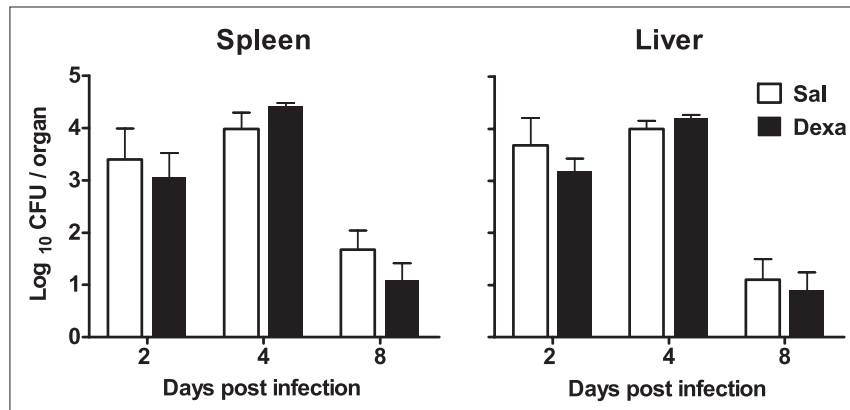

FIGURE 8 | No differences in susceptibility to L. monocytogenes infection were observed between Sal and Dexa rats. The bacterial loads of Sal and Dexa rats infected with $L$. monocytogenes were determined at 2, 4, and 8 days post infection in spleen and liver. Data are represented as the mean + SEM from seven to nine rats per group.

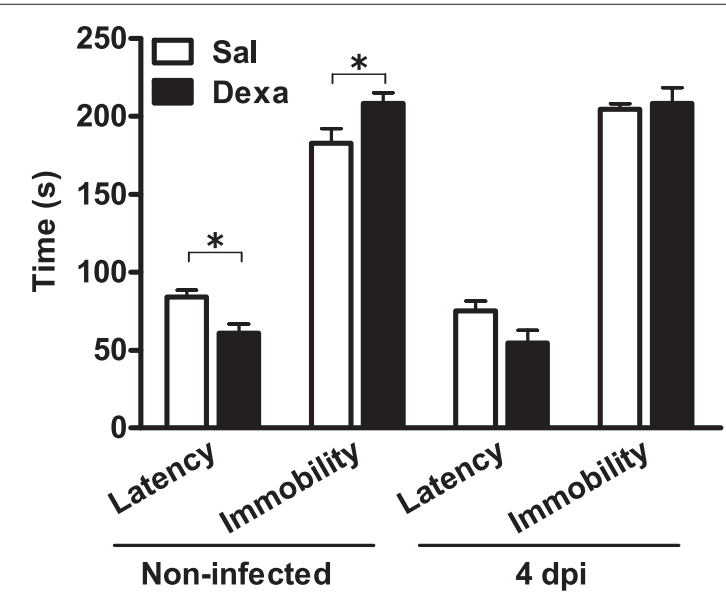

FIGURE 9 | Infection with $L$. monocytogenes does not aggravate the depressive-like behavior of Dexa animals. Latency to first immobility and duration of immobility in the forced swim test were assessed in Sal and Dexa rats non-infected and 4 days after infection with $L$. monocytogenes ( 4 dpi). Each bar represents the mean + SEM from seven to nine rats per group.

the formation of regulatory T cells. However, this is the first study showing that prenatal dexamethasone treatment can induce, in adulthood, alterations in the proportion of $\mathrm{CD}^{+}$regulatory $\mathrm{T}$ cells, a subtype of cells of crucial relevance not only in the immune system response, but also in the modulation of depressive-like behavior (Rook and Lowry, 2008; Miller, 2010). A final note to the fact that B cells were also increased in the spleen of adult rats prenatally treated with dexamethasone. Since, in rodents, B cells immunoglobulins expression appears in the liver, spleen, and bone marrow at day 17 of gestation (Holladay and Smialowicz, 2000), it seems reasonable to suggest that dexamethasone administration on days 18 and 19 of gestation also targeted the function of $\mathrm{B}$ cells. Interestingly, we observed a negative correlation between the percentage of $\mathrm{CD} 8^{+} \mathrm{T}$ cells and the latency time in the FST, and an almost positive correlation between the percentage of B cells and the same behavioral parameter. These correlations suggest that alterations in depressive-like behavior may be associated with differences in the percentage of specific immune cells populations, which should next be further investigated.
The most likely mediators of the cross-talk between the immune and the nervous systems are cytokines. Several studies have demonstrated their role in the pathophysiology of depression (Miller et al., 2009). Since we showed that prenatal dexamethasone administration can induce alterations in adults' immune cells we also analyzed the production of cytokines. In basal conditions, a tendency for an overall decrease in the production of all cytokines analyzed in the serum was observed, but only IL-6 and IL-10 levels were significantly reduced. It is important to note that the anti-inflammatory cytokine IL-10 has been shown to play a major role in depression. In fact, the absence of IL-10 is able to induce, in mice, depressive-like behavior and IL-10 administration rescues this phenotype (Mesquita et al., 2008). Moreover, due to the interplay between IL-10 and glucocorticoids, it has been suggested that this anti-inflammatory cytokine can be a putative link between disruption of the HPA axis and the imbalanced production of cytokines observed in depressed patients (Roque et al., 2009). Accordingly, in this distinct rat model, we found: (i) disruption of the HPA axis (Oliveira et al., 2006); (ii) decreased levels of IL-10; and (iii) depressive-like behavior.

Assessment of basal conditions in regulatory systems, such as the nervous and the immune systems, can only provide a "one-side of the coin perspective". In fact, the ability of these regulatory systems to tackle with challenges is critical for their evaluation. Our present data certainly confirms this perspective. After a challenge with LPS, spleen cells from rats prenatally treated with dexamethasone respond with an increased production of IL-6. Taking into account that LPS is recognized by Toll-like receptor 4 (TLR4), this result shows that there is an over reactive innate response to this stimulus. Of notice, there is evidence that dexamethasone can increase the expression of TLR4 (Galon et al., 2002). Thus, pre-exposure to dexamethasone has been shown to enhance LPS responsiveness (Barber et al., 1993; Zhang and Daynes, 2007). Irrespectively from the underlying mechanism, the present data show an increased pro-inflammatory profile in response to LPS in animals prenatally treated with dexamethasone, which fit previous observations by Bakker et al. (2000) in rodents, but also a human study that revealed that premature newborns treated with dexamethasone present, at school age, a shift toward a more pro-inflammatory cytokine profile (Karemaker et al., 2008).

Our next step was to perform a multimodal analysis after a combined innate/acquired immune stimulus through infection with L. monocytogenes. $\mathrm{CD}^{+} \mathrm{T}$ cells, which we found decreased in prenatally treated Dexa rats, play a major role in the immune response to L. monocytogenes. After acute infection, the differences observed in the proportion of spleen $\mathrm{CD} 8^{+} \mathrm{T}$ cells between non-infected Dexa and Sal animals disappeared. Moreover, the proportion of all spleen cell populations became identical between dexamethasone- and vehicle-exposed rats. This suggests that despite an altered homeostasis of the immune system, Dexa animals can mount an adequate immune response to L. monocytogenes infection. In accordance, no changes were found in the serum cytokine profile. It is therefore not surprising that the bacterial load in both groups of animals was also similar. Altogether, prenatal dexamethasone treatment does not cause alterations in susceptibility to acute infection with this Grampositive bacterium. Of relevance, we observed that $L$. monocytogenes infection in Sal animals triggered a trend for depressive-like behavior $(P=0.06)$, which fits the hypothesis that the increased production of pro-inflammatory cytokines is linked to alterations in emotional 
behavior. As Dexa rats already presented depressive-like phenotype in basal conditions, we did not observe a significant aggravation of this behavior phenotype.

In conclusion, we herein show that prenatal administration of dexamethasone induces, in adulthood, depressive-like behavior and immune system alterations. Additional studies should next address which are the precise mediators of such interplay.

\section{REFERENCES}

Ashwell, J. D., Lu, F. W., and Vacchio, M. S. (2000). Glucocorticoids in T cell development and function. Annu. Rev. Immunol. 18, 309-345.

Bakker, J. M., Kavelaars, A., Kamphuis, P. J., Cobelens, P. M., van Vugt, H. H., van Bel, F., and Heijnen, C. J. (2000). Neonatal dexamethasone treatment increases susceptibility to experimental autoimmune disease in adult rats. J. Immunol. 165, 5932-5937.

Bakker, J. M., Kavelaars, A., Kamphuis, P. J., Zijlstra, J., van Bel, F., and Heijnen, C. J. (2001). Neonatal dexamethasone treatment induces long-lasting changes in T-cell receptor vbeta repertoire in rats. J. Neuroimmunol. 112, 47-54.

Bakker, J. M., Schmidt, E. D., Kroes, H., Kavelaars, A., Heijnen, C. J., Tilders, F. J., and van Rees, E. P. (1995). Effects of short-term dexamethasone treatment during pregnancy on the development of the immune system and the hypothalamo-pituitary adrenal axis in the rat. J. Neuroimmunol. 63, 183-191.

Barber, A. E., Coyle, S. M., Marano, M. A., Fischer, E., Calvano, S. E., Fong, Y., Moldawer, L. L., and Lowry, S. F. (1993). Glucocorticoid therapy alters hormonal and cytokine responses to endotoxin in man. J. Immunol. 150, 1999-2006.

Bessa, J.M., Ferreira, D., Melo, I., Marques, F., Cerqueira, J. J., Palha, J.A., Almeida, O. F., and Sousa, N. (2009). The moodimproving actions of antidepressants do not depend on neurogenesis but are associated with neuronal remodeling. Mol. Psychiatry 14, 764-773, 739.

Cabanes, D., Lecuit, M., and Cossart, P. (2008). Animal models of Listeria infection. Curr. Protoc. Microbiol. Chapter 9, Unit9B.1.

Coe, C. L., and Lubach, G. R. (2000). Prenatal influences on neuroimmune set points in infancy. Ann. N. Y. Acad. Sci. 917, 468-477.

Coe, C. L., and Lubach, G. R. (2005). Developmental consequences of antenatal dexamethasone treatment in nonhuman primates. Neurosci. Biobehav. Rev. 29, 227-235.

Dietert, R. R., Lee, J. E., Olsen, J., Fitch, K., and Marsh,J.A. (2003). Developmental immunotoxicity of dexamethasone: comparison of fetal versus adult exposures. Toxicology 194, 163-176.
Galon, J., Franchimont, D., Hiroi, N., Frey, G., Boettner, A., Ehrhart-Bornstein, M., O'Shea, J. J., Chrousos, G. P., and Bornstein, S. R. (2002). Gene profiling reveals unknown enhancing and suppressive actions of glucocorticoids on immune cells. FASEB J. 16, 61-71.

Goettsch, W., Garssen, J., de Klerk, A., Herremans, T. M., Dortant, P., de Gruijl, F. R., and Van Loveren, H. (1996). Effects of ultraviolet-B exposure on the resistance to Listeria monocytogenes in the rat. Photochem. Photobiol. 63, 672-679.

Goncalves, L., Silva, R., Pinto-Ribeiro, F., Pego, J. M., Bessa, J. M., Pertovaara, A., Sousa, N., and Almeida, A. (2008). Neuropathic pain is associated with depressive behaviour and induces neuroplasticity in the amygdala of the rat. Exp. Neurol. 213, 48-56.

Hauser, J., Feldon, J., and Pryce, C. R. (2009). Direct and dam-mediated effects of prenatal dexamethasone on emotionality, cognition and HPA axis in adult Wistar rats. Horm. Behav. 56, 364-375.

Hirvikoski, T., Nordenstrom, A., Lindholm, T., Lindblad, F., Ritzen, E. M., Wedell, A., and Lajic, S. (2007). Cognitive functions in children at risk for congenital adrenal hyperplasia treated prenatally with dexamethasone. J. Clin. Endocrinol. Metab. 92, 542-548.

Hofmeyr, G. J. (2009). Antenatal Corticosteroids for Women at Risk of Preterm Birth: RHL Commentary. Geneva: The WHO Reproductive Health Library, World Health Organization.

Holladay, S. D., and Smialowicz, R. J. (2000). Development of the murine and human immune system: differential effects of immunotoxicants depend on time of exposure. Environ. Health Perspect. 108(Suppl. 3), 463-473.

Karagiannidis, C., Akdis, M., Holopainen, P., Woolley, N. J., Hense, G., Ruckert, B., Mantel, P. Y., Menz, G., Akdis, C. A., Blaser, K., and Schmidt-Weber, C. B. (2004). Glucocorticoids upregulate Foxp3 expression and regulatory T cells in asthma. J. Allergy Clin. Immunol. 114, 1425-1433.

Karemaker, R., Kavelaars, A., ter Wolbeek, M., Tersteeg-Kamperman, M., Baerts, W., Veen, S., Samsom, J. F., Visser, G.

\section{ACKNOWLEDGMENTS}

We acknowledge the Portuguese Foundation for Science and Technology (FCT) for providing a fellowship to Susana Roque (SFRH/BD/24539/2005), Claudia Nobrega (SFRH/ BPD/65380/2009), and Cláudio Nunes-Alves (SFRH/ $\mathrm{BD} / 38614 / 2007)$. This work was also supported by a FCT grant PTDC/SAU-NEU/105180/2008.

H., van Bel, F., and Heijnen, C. J. (2008). Neonatal dexamethasone treatment for chronic lung disease of prematurity alters the hypothalamuspituitary-adrenal axis and immune system activity at school age. Pediatrics 121, e870-e878.

Leao, P., Sousa, J. C., Oliveira, M., Silva, R., Almeida, O. F., and Sousa, N. (2007). Programming effects of antenatal dexamethasone in the developing mesolimbic pathways. Synapse 61, 40-49.

Mesquita,A.R., Correia-Neves, M., Roque, S., Castro, A. G., Vieira, P., Pedrosa, J., Palha, J.A., and Sousa, N. (2008). Il-10 modulates depressive-like behavior. $J$. Psychiatr. Res. 43, 89-97.

Mesquita, A. R., Wegerich, Y., Patchev, A. V., Oliveira, M., Leao, P., Sousa, N., and Almeida, O. F. (2009). Glucocorticoids and neuro- and behavioural development. Semin. Fetal Neonatal Med. 14, 130-135.

Miller, A. H. (2010). Depression and immunity: a role for T cells? Brain Behav. Immun. 24, 1-8.

Miller, A. H., Maletic, V., and Raison, C. L. (2009). Inflammation and its discontents: the role of cytokines in the pathophysiology of major depression. Biol. Psychiatry 65, 732-741.

Oliveira, M., Bessa, J. M., Mesquita, A., Tavares, H., Carvalho, A., Silva, R., Pego, J. M., Cerqueira, J. J., Palha, J. A., Almeida, O. F., and Sousa, N. (2006). Induction of a hyperanxious state by antenatal dexamethasone: a case for less detrimental natural corticosteroids. Biol. Psychiatry 59, 844-852.

Oliveira, M., Leão, P., Rodrigues, A.-J., Pêgo, J.-M., Cerqueira, J.-J., and Sousa, N. (2010). Programming effects of antenatal corticosteroids exposure in male sexual behavior. J. Sex. Med. doi: 10.1111/j.1743-6109.2010.02170.x. [Epub ahead of print].

Pamer, E. G. (2004). Immune responses to Listeria monocytogenes. Nat. Rev. Immunol. 4, 812-823.

Rodrigues, A.-J., Leão, P., Oliveira, M., Pêgo, J.-M., Franky,A., Morgado, P., Mesquita, A.,Araujo, D.,Almeida, O.F., and Sousa., N. (2010)."Vulnerability toaddiction can be modulated by dopamine," in Poster Presented at Society for Neurosciences, November 13-17, San Diego.

Rook, G. A., and Lowry, C. A. (2008). The hygiene hypothesis and psychiatric disorders. Trends Immunol. 29, 150-158.

Roque, S., Correia-Neves, M., Mesquita,A. R., Palha, J. A., and Sousa, N. (2009). Interleukin-10: a key cytokine in depression? Cardiovasc. Psychiatry Neurol. 2009, 187894.

Sultzer, B. M., and Goodman, G. W. (1976). Endotoxin protein: a B-cell mitogen and polyclonal activator of C3h/Hej lymphocytes. J. Exp. Med. 144, 821-827.

Trautman, P. D., Meyer-Bahlburg, H. F., Postelnek, J., and New, M. I. (1995). Effects of early prenatal dexamethasone on the cognitive and behavioral development of young children: results of a pilot study. Psychoneuroendocrinology 20, 439-449.

Welberg, L. A., and Seckl, J. R. (2001). Prenatal stress, glucocorticoids and the programming of the brain. J. Neuroendocrinol. 13, 113-128.

Zhang, T. Y., and Daynes, R. A. (2007). Glucocorticoid conditioning of myeloid progenitors enhances Tlr4 signaling via negative regulation of the phosphatidylinositol 3-kinaseAkt pathway. J. Immunol. 178, 2517-2526.

Conflict of Interest Statement: The authors declare that the research was conducted in the absence of any commercial or financial relationships that could be construed as a potential conflict of interest.

Received: 17 August 2010; accepted: 19 January 2011; published online: 07 February 2011.

Citation: Roque S, Oliveira TG, Nobrega C, Barreira-Silva P, Nunes-Alves C, Sousa N, Palha JA and Correia-Neves M (2011) Interplay between depressive-like behavior and the immune system in an animal model of prenatal dexamethasone administration. Front. Behav. Neurosci. 5:4. doi: 10.3389/ fnbeh.2011.00004

Copyright (C) 2011 Roque, Oliveira, Nobrega, Barreira-Silva, Nunes-Alves, Sousa, Palha and Correia-Neves. This is an open-access article subject to an exclusive license agreement between the authors and Frontiers Media SA, which permits unrestricted use, distribution, and reproduction in any medium, provided the original authors and source are credited. 